\title{
Predictores de la Intención Emprendedora en Alumnos de Pregrado: Un Modelo Basado en Ecuaciones Estructurales
}

\author{
Carlos Galleguillos-Cortés ${ }^{(1,2) \star}$, Braian Escobar-Burgos ${ }^{(2)}$ y Rubén Hurtado-Cailly ${ }^{(3)}$ \\ (1) Facultad de Administración y Economía, Departamento de Administración, Universidad de Santiago de \\ Chile, Santiago, Chile. (e-mail: carlos.galleguillos@usach.cl) \\ (2) Facultad de Ingeniería, Departamento de Industria y Negocios, Universidad de Atacama, Copiapó, Chile. \\ (e-mail: carlos.galleguillos@uda.cl) \\ (3) Facultad Tecnológica, Departamento de Tecnologías de la Administración y Gestión, Universidad de \\ Atacama, Copiapó, Chile.
}

* Autor a quien debe ser dirigida la correspondencia

Recibido Jun. 1, 2018; Aceptado Ago. 1, 2018; Versión final Ago. 20, 2018, Publicado Feb. 2019

\begin{abstract}
Resumen
Se ha determinado los factores que afectan la intención emprendedora de alumnos de pregrado mediante la adaptación de la teoría del comportamiento planificado y modelado a través de ecuaciones estructurales. Esta teoría se usa para identificar las creencias conductuales, normativas y de control, pertinentes a la intención emprendedora. La hipótesis de investigación es que la intención se predice por la creencia sobre la aversión al riesgo, la disposición respecto a personas importantes y la autoeficacia. El modelo es probado a través de una encuesta de 1.339 alumnos de las facultades de ingeniería, salud, humanidades, jurídicas y tecnológica, en una universidad pública y acreditada en Chile. Los resultados confirman las hipótesis planteadas. Esto es que la actitud, la norma subjetiva y el control de la conducta percibida son significativas y están positivamente relacionadas con la intención emprendedora.
\end{abstract}

Palabras clave: intención emprendedora; teoría del comportamiento planificado; ecuaciones estructurales

\section{Predictors of Entrepreneurial Intention in Undergraduate Students: A Model Based on Structural Equations}

\begin{abstract}
.
This research aims to determine the factors that affect the entrepreneurial intention of undergraduate students by adapting the theory of planned behavior and modeled through structural equations. This theory is used to identify the behavioral, normative and control beliefs pertinent to the entrepreneurial intention. The hypothesis of the research model is that the intention is predicted by the belief about risk aversion, the disposition regarding important people and self-efficacy. The research model was tested through a survey of 1,339 students from the faculties of engineering, health, humanities, law and technology, in a public and accredited university in Chile. The results confirm the hypotheses proposed. This means that the attitude, the subjective norm and the control of the perceived behavior are significant and positively related to the entrepreneurial intention.
\end{abstract}

Keywords: entrepreneurial intention; theory of planned behavior; structural equations 


\section{INTRODUCCIÓN}

La intención emprendedora ha recibido una considerable atención por parte de los investigadores, principalmente por su contribución al crecimiento económico y aumento de la empleabilidad (Shane y Venkataraman, 2000) a través de la capacidad de crear nuevas empresas y la innovación (Teixeira et al, 2018). En este sentido, el emprendimiento definido como la capacidad de crear o identificar oportunidades comerciales y explorarlas, con el fin de crear valor y beneficio (Shane y Venkataraman, 2000), involucra competencias como la dedicación, esfuerzo y sobre todo la capacidad de asumir riesgos financieros, psicológicos y sociales para obtener lo deseado. Dado este enfoque, las universidades son las llamadas a desempeñar un papel más activo, en particular proporcionando a sus estudiantes competencias que facilitan la realización de una carrera emprendedora. Así, la implicancia de las universidades es tanto más importante puesto que la intención emprendedora se está convirtiendo en una competencia más común y necesaria para los estudiantes en el siglo XXI, (Iglesias-Sánchez et al, 2016).

Como resultado de lo anteriormente señalado, ha surgido el prominente interés por estudiar los predictores de las intenciones emprendedoras (Fietze y Boyd 2017; Entrialgo e Iglesias, 2016), puesto que son clave para entender el emprendimiento, ya que la creencia, el deseo de iniciar o poseer un negocio se describe a través de estos (Krueger et al., 2000). Sin embargo, a pesar del interés y la relevancia que tiene el estudio de las intenciones emprendedoras, se han encontrado hallazgos empíricos inconclusos en cuanto a los resultados de las relaciones entre la intención emprendedora y sus variables independientes (Alferaih, 2017)

En este contexto, el estudio tiene como objetivo evaluar empíricamente los determinantes de la intención emprendedora de alumnos de pregrado mediante la adaptación de la teoría del comportamiento planificado. Además, los resultados obtenidos son útiles para comprender y comparar el efecto de las variables respecto a países e intenciones empresariales.

\section{Educación para el desarrollo del emprendimiento}

El emprendimiento se puede definir como una actividad que involucra el descubrimiento, la evaluación, y la explotación de oportunidades que reúne factores productivos para realizar el proceso de producción y que ocurre a lo largo del tiempo (Liñán y Chen, 2009). Se argumenta que la educación empresarial formal temprana podría afectar a los estudiantes en sus actitudes, influir en la dirección de su carrera futura y afectar su propensión para el emprendimiento cuando se hacen adultos. Así mismo, se cree que este tipo de educación brinda: habilidades generales a partir de la capacitación y conocimiento, facilita el acceso al mundo de los negocios al permitir que las personas evalúen la extensión del mercado laboral, el tipo de bienes que demandan los clientes y la organización del negocio (Rauch y Hulsink 2015). No obstante, evidencia contradictoria aumenta la incertidumbre sobre la efectividad de los programas académicos (Soria-Barreto et al. 2016; Lima, et al, 2015). Por ejemplo, principalmente aún existe dificultades con la metodología (Westhead et al., 2001). Esto presenta claramente problemas para los investigadores y complica aún más el debate que rodea si se puede enseñar o no el emprendimiento.

\section{Intención emprendedora}

La decisión de convertirse en emprendedor es una decisión premeditada y consciente. Es decir, es el estado mental consciente que precede a la acción que dirige la atención hacia un objetivo, como por ejemplo comenzar un nuevo negocio, (Krueger et al, 2000). Un meta-análisis previo sobre la intención emprendedora identifico setenta y dos variables independientes que han sido estudiada, (Alferaih, 2017). En general, estas se pueden agrupar en tres focos que predominan al momento de determinar quién puede ser un emprendedor. Primero, el perfil demográfico que incluye edad, sexo, experiencia previa (Yukongdi, y Lopa 2017). Segundo, los factores contextuales que incluye educación, medio ambiente y cultura (Solesvik, 2014). Finalmente, en tercer lugar, investigaciones presentan a las características de la personalidad como identificadores de los empresarios, estas incluyen la autoeficacia, la confianza, autonomía, locus de control, tendencia a asumir riesgos, atracción profesional. Sin embargo, respecto a las variables más utilizadas según Alferaih, A, los resultados han sido inconsistente y abierta a la crítica entre teóricos e investigadores, (Alferaih, 2017; Kirkley 2016).

En la última década, a nivel latinoamericano, los estudios de la intención emprendedora se concentran principalmente en tres países, Chile, Colombia y Uruguay. La tabla 1 resumen las evidencias encontradas, identificando una diversidad de enfoques metodológicos, en términos de propósitos, teoría, análisis estadísticos (regresión logística, ANOVA, ecuaciones estructurales), tamaño de muestra (entre 62 a 1088 estudiantes) y tamaños de los efectos, lo que impide hacer comparaciones. 
Tabla 1: Breve revisión de la literatura a nivel latinoamericano.

\begin{tabular}{|c|c|c|c|}
\hline Autor & Titulo & Metodología & Conclusión \\
\hline $\begin{array}{l}\text { Krauss, C., Franco, } \\
\text { J. P., Bonomo, A., } \\
\text { Mandirola, N., y } \\
\text { Platas, A. L. (2018). }\end{array}$ & $\begin{array}{l}\text { Intención emprendedora en } \\
\text { estudiantes de ciencias de la } \\
\text { salud de la Universidad Católica } \\
\text { del Uruguay }\end{array}$ & $\begin{array}{l}1.088 \text { estudiantes } \\
\text { Análisis cuantitativo } \\
\text { Asociaciones } \\
\text { estadísticas (pruebas chi- } \\
\text { cuadrado) y diferencia de } \\
\text { medias significativas }\end{array}$ & $\begin{array}{l}\text { La IE de estudiantes de } \\
\text { Ciencias de la Salud es menor } \\
\text { respecto al resto de los } \\
\text { estudiantes de dicha } \\
\text { Universidad. }\end{array}$ \\
\hline $\begin{array}{l}\text { Tarapuez, E., } \\
\text { García, M. D., y } \\
\text { Castellano, N. } \\
\text { (2018). }\end{array}$ & $\begin{array}{l}\text { Aspectos socioeconómicos e } \\
\text { intención emprendedora en } \\
\text { estudiantes universitarios del } \\
\text { Quindío (Colombia) }\end{array}$ & $\begin{array}{l}297 \text { estudiantes } \\
\text { Análisis cuantitativo } \\
\text { Correspondencias } \\
\text { Múltiples } \\
\text { Regresión Logística }\end{array}$ & $\begin{array}{l}\text { Se encontró que los dos } \\
\text { aspectos socioeconómicos } \\
\text { que más influyen en la IE de los } \\
\text { estudiantes universitarios son } \\
\text { el "tipo de universidad" y "tener } \\
\text { un amigo empresario". }\end{array}$ \\
\hline $\begin{array}{l}\text { Laguía, A., Moriano, } \\
\text { J. A., Molero, F., y } \\
\text { Gámez, J. A. } \\
\text { (2017). }\end{array}$ & $\begin{array}{l}\text { Validación del Cuestionario de } \\
\text { Intención Emprendedora en una } \\
\text { muestra de estudiantes } \\
\text { universitarios de Colombia. }\end{array}$ & $\begin{array}{l}316 \text { estudiantes } \\
\text { Análisis cuantitativo } \\
\text { Regresión logística }\end{array}$ & $\begin{array}{l}\text { Un mejor conocimiento de los } \\
\text { antecedentes psicosociales } \\
\text { que conducen a jóvenes } \\
\text { universitarios a la IE. }\end{array}$ \\
\hline $\begin{array}{l}\text { Tarapuez } \\
\text { Chamorro, E. } \\
(2016)\end{array}$ & $\begin{array}{l}\text { Las dimensiones culturales de } \\
\text { Geert Hofstede y la intención } \\
\text { emprendedora en estudiantes } \\
\text { universitarios del departamento } \\
\text { del Quindío (Colombia) }\end{array}$ & $\begin{array}{l}297 \text { estudiantes } \\
\text { Análisis cuantitativo } \\
\text { Regresión logística }\end{array}$ & $\begin{array}{l}\text { Los jóvenes de las } \\
\text { universidades privadas están } \\
\text { más asociados con una baja IE } \\
\text { y con el hecho de tener o haber } \\
\text { tenido una empresa, en tanto } \\
\text { que los de la universidad } \\
\text { pública están más } \\
\text { relacionados con una seria IE. }\end{array}$ \\
\hline $\begin{array}{l}\text { Soria-Barreto, K., } \\
\text { Zuniga-Jara, S., y } \\
\text { Ruiz-Campo, S. } \\
(2016)\end{array}$ & $\begin{array}{llr}\text { Educación } & \text { e Intención } \\
\text { Emprendedora en Estudiantes } & \text { en En } \\
\text { Universitarios: Un Caso de } \\
\text { Estudio }\end{array}$ & $\begin{array}{l}62 \text { estudiantes. } \\
\text { Análisis cuantitativo } \\
\text { ANOVA }\end{array}$ & $\begin{array}{l}\text { Los resultados del estudio } \\
\text { muestran que la IE fue } \\
\text { afectada negativamente por el } \\
\text { curso impartido. }\end{array}$ \\
\hline $\begin{array}{l}\text { Osorio, F. F., y } \\
\text { Londoño Roldán, J. } \\
\text { C. (2015). }\end{array}$ & $\begin{array}{l}\text { Intención emprendedora de } \\
\text { estudiantes de educación media: } \\
\text { extendiendo la teoría de } \\
\text { comportamiento planificado } \\
\text { mediante el efecto exposición }\end{array}$ & $\begin{array}{l}643 \text { estudiantes } \\
\text { Análisis cuantitativo } \\
\text { Ecuaciones estructurales. }\end{array}$ & $\begin{array}{l}\text { Los resultados muestran que la } \\
\text { exposición previa en } \\
\text { actividades de emprendimiento } \\
\text { tiene un efecto positivo sobre } \\
\text { los determinantes de la IE. }\end{array}$ \\
\hline
\end{tabular}

Dado lo anterior, sería más apropiado para contribuir a la comparación de los resultados, el uso de escalas frecuentemente utilizadas, las que a su vez interpreten de mejor forma la intención emprendedora, en este sentido la presente investigación se apoya en la Teoría del Comportamiento Planificado.

\section{Teoría del Comportamiento Planificado}

La Teoría del Comportamiento Planificado, en adelante (TPB), se considera adecuada en el contexto del emprendimiento porque este es un comportamiento que está bajo control voluntario. Este modelo, analiza las creencias del individuo traducidas en las actitudes personales (A), las normas sociales (SN) y el control conductual percibido (PBC), como causas de la intención, la cual a su vez influyen en el comportamiento (Azjen, 1991). En otras palabras, son creencias correspondientes, que refleja la estructura cognitiva subyacente. Cada creencia de comportamiento vincula un actuar determinado a un resultado determinado, o a algún otro atributo, como el costo incurrido en la realización del comportamiento (Armitage y Conner, 2001).

La intención se ha empleado ampliamente para comprender los problemas sociales y aplicados respecto al comportamiento. Por ejemplo, de acuerdo con el meta-análisis de (Sheeran, P, 2002) que cubre 422 estudios durante 10 años, indicó que las intenciones explican el $28 \%$ de la varianza, en promedio, en el comportamiento futuro del individuo. Investigaciones afirman que la intención emprendedora es un indicador de la voluntad de los individuos de intentar, el nivel de esfuerzo y la disposición para participar en ciertos comportamientos. Por lo tanto, la intención emprendedora es la representación cognitiva de la voluntad de los individuos para comportarse de ciertas maneras (Fayolle y Gailly 2015), la cual ha sido reconocida como la propensión a ser innovadora, proactiva, abierta al riesgo, autónoma y agresivamente competitiva.

En general, estudios han utilizado la teoría para explicar las intenciones de convertirse en emprendedor (Krueger et al., 2000; Liñán y Chen, 2009) y el comportamiento empresarial (Kautonen, et al, 2013), así como 
los efectos de la educación empresarial (Liñán et al., 2011). Un meta-análisis que evalúa la (TPB) en el contexto de la iniciativa empresarial informó que las actitudes, las normas subjetivas y el control conductual percibido representaron 39\% de la variación en las intenciones empresariales (Schlaegel y Koenig, 2011). Adicionalmente, (Kautonen, et al, 2013) proporcionó evidencia sobre la predicción de las intenciones y comportamiento respecto a la puesta en marcha de empresas basado en datos longitudinales (2006 y 2009) en una población en edad de trabajar en Finlandia. Los resultados apoyan la predicción resumida en el (TPB), es decir la actitud, las normas subjetivas y el control percibido del comportamiento son predictores significativos de la intención empresarial. A su vez, la intención y el control conductual percibido son significativos predictores de comportamiento posterior. Otras investigaciones han respaldado la validez predictiva de las intenciones sobre los comportamientos cuando son influenciadas por la educación empresarial (Bae et al, 2014).

Actualmente, evidencia en torno al emprendimiento social han mostrado una relación positiva y significativa entre el control conductual percibido y la intención emprendedora (Al-Shammari, 2018). Además, hubo una importante relación positiva moderada entre las normas subjetivas percibidas por los estudiantes y la valoración del emprendimiento social con su intención emprendedora. Por el contrario, existe evidencia indirecta contradictoria sobre la influencia de algunos de los predictores de la intención. Según (Sitaridis y Kitsios 2017), quienes investigaron la influencia de las intenciones empresariales de los estudiantes de tecnología de la información (TI). Los investigadores encontraron que las actitudes personales y el control conductual percibido demostraron jugar un papel vital en las intenciones empresariales, mientras que la norma social no tuvo un efecto significativo. Además, en el mismo estudio, concluyeron que las estudiantes mostraron una menor intención hacia la iniciativa empresarial, en comparación con sus homólogos masculinos.

En consecuencia, el objetivo de esta investigación es determinar los factores que influyen en la intención a emprender en alumnos de pregrado en una universidad pública y acreditada en Chile, apoyado en una teoría propicia para predecir como lo es la Teoría del Comportamiento Planificado, la cual supone que un comportamiento se explica mejor por la intención de intentar realizar el comportamiento (Azjen, 1991).

\section{HIPÓTESIS DE TRABAJO}

En primer lugar, las actitudes están determinadas por las creencias de que cierto comportamiento conducirá a un resultado favorable. Por ejemplo, en algunas investigaciones se ha medido el compromiso con los nuevos negocios y la voluntad de sacrificar recurso para el desarrollo de iniciativas empresariales basado en la creencia de asumir riesgos. Luego, en el contexto de esta investigación se desea probar si la creencia fundamentada en el asumir riesgos influye en la intención a emprender en los estudiantes universitario de pregrado. Es decir, el grado en que el alumno amplía los límites para poner a prueba sus ideas e iniciativas en un entorno desconocido. H1: Una actitud basada en la aversión al riesgo está positiva y significativamente relacionada con intención emprendedora.

En segundo lugar, las normas subjetivas están determinadas por las creencias de otros relevantes (amigos, familia, compañeros de trabajo) sobre un determinado comportamiento y el grado en que tienden a cumplir con estas creencias, (Ajzen, 1991). En otras palabras, mide el valor que las personas asignan a la opinión de otras personas cercanas a ellos. Se sospecha que, al tomar una iniciativa empresarial, los estudiantes valoran la opinión de la familia, amigos y compañeros de curso. Por tanto, se desea probar la influencia que tiene la familia, los amigos y los compañeros de curso sobre la intención a emprender. H2: Las creencias normativas están positiva y significativamente relacionada con intención emprendedora.

Finalmente, el control conductual percibido refleja las percepciones sobre el comportamiento como algo personalmente controlable, así como las nociones relacionadas con la facilidad o dificultad de iniciar un comportamiento. En este sentido, la autoeficacia cobra relevancia, puesto que dicho constructo permite movilizar a las personas sobre la base de sus capacidades y la confianza en sí mismo. Estudios previos han confirmado una relación positiva y significativa, (Thomas et al., 2014; Kautonen, et al, 2013). Por tanto, se desea probar si la creencia que tiene el alumno de poseer las capacidades para desempeñar las acciones necesarias que le permitan obtener los resultados deseados, en términos de la confianza en sí mismo, está relacionado con la intención emprendedora. H3: El control conductual percibido, basado en la autoeficacia está positivamente relacionado con la intención emprendedora.

De esta forma, el modelo conceptual hipotetizado es el que muestra la Figura 1: 


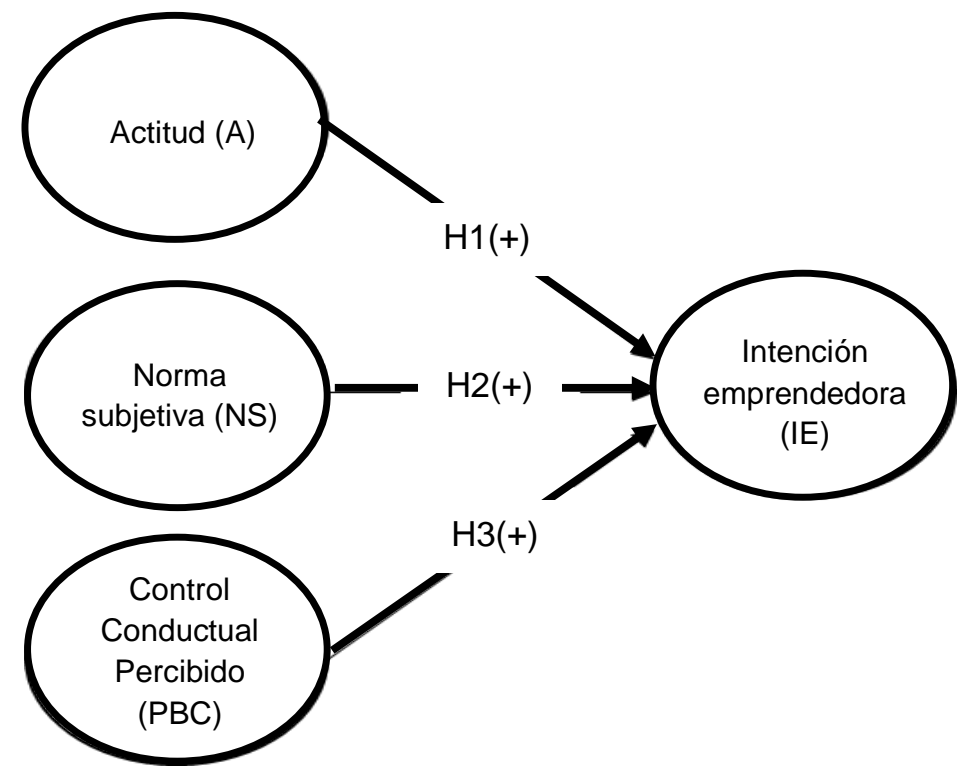

Fig. 1: Modelo conceptual hipotetizado.

\section{METODOLOGÍA}

Esta sección presenta el proceso metodológico sugerido, que permite alcanzar el objetivo definido, es decir medir los determinantes de la intención emprendedora en los estudiantes de pregrado, basado en la (TPB). Donde la variable dependiente es la intención emprendedora y las variables independientes son las actitudes personales (A), las normas subjetivas (SN) y el control conductual percibido (PBC).

\section{Muestra}

La muestra consistió en 1.339 estudiantes de educación superior matriculados en carreras diurnas del primer semestre del año académico 2017 en una universidad pública y acreditada en Chile. La muestra se distribuye según las facultades de ingeniería (24,9\%), salud (20.2\%), humanidades (18.7\%), jurídicas (17.4\%) y tecnológica (18.8\%). La edad promedio fue de 21 años. Respecto al género, la tabla 1 informa que se encuestaron 558 correspondiente al $(41,7 \%)$ de estudiantes de sexo masculino y 781 equivalente $(58.3 \%)$ de sexo femenino. De igual manera la tabla 3 señala la distribución de la muestra según el sexo respecto al nivel cursado, donde se evidencia el predominio del sexo femenino desde el 1er al 5 to año.

Tabla 2: Distribución de la muestra de la facultad respecto al sexo.

\begin{tabular}{|l|c|c|c|}
\hline \multicolumn{1}{|c|}{ Facultad } & Femenino & Masculino & Total \\
\hline Cs Jurídicas y Sociales & 151 & 82 & 233 \\
\hline Cs de la Salud & 228 & 43 & 271 \\
\hline Humanidades y Educación & 197 & 54 & 251 \\
\hline Cs de la Ingeniería & 127 & 207 & 334 \\
\hline Tecnológica & 78 & 172 & 250 \\
\hline Total & 781 & 558 & 1.339 \\
\hline
\end{tabular}

Tabla 3: Distribución de la muestra según el sexo respecto al nivel cursado.

\begin{tabular}{|c|c|c|c|c|c|c|c|}
\hline Sexo & $1^{\circ}$ Año & $2^{\circ}$ Año & $3^{\circ}$ Año & $4^{\circ}$ Año & $5^{\circ}$ Año & $6^{\circ}$ Año & Total \\
\hline Femenino & 250 & 265 & 153 & 60 & 39 & 14 & 781 \\
\hline Masculino & 208 & 168 & 108 & 36 & 19 & 19 & 558 \\
\hline Total & 458 & 433 & 261 & 96 & 58 & 33 & 1.339 \\
\hline
\end{tabular}

La tabla 4 presenta un resumen sobre la distribución entre la Facultad y nivel cursado. Existe una concentración de alumnos en los tres primeros niveles, con una proporción del $86 \%$. Esto ocurre debido a que las carreras tienen en promedio ente 4 a 5 años de duración. Las únicas especialidades que extienden ese plazo son las relacionadas con la Facultad de Ingeniería donde el tiempo es de 6 años. 
Tabla 4: Descripción de Facultad según nivel.

\begin{tabular}{|l|c|c|c|c|c|c|c|}
\hline \multicolumn{1}{|c|}{ Facultad } & $1^{\circ}$ Año & $2^{\circ}$ Año & $3^{\circ}$ Año & $4^{\circ}$ Año & $5^{\circ}$ Año & $6^{\circ}$ Año & Total \\
\hline Cs Jurídicas y Sociales & 98 & 51 & 63 & 11 & 10 & 0 & 233 \\
\hline Cs de la Salud & 133 & 75 & 26 & 24 & 13 & 0 & 271 \\
\hline Humanidades y Educación & 53 & 104 & 62 & 23 & 9 & 0 & 251 \\
\hline Cs de la Ingeniería & 50 & 120 & 68 & 37 & 26 & 33 & 334 \\
\hline Tecnológica & 124 & 83 & 42 & 1 & 0 & 0 & 250 \\
\hline \multicolumn{1}{|c|}{ Total } & 458 & 433 & 261 & 96 & 58 & 33 & 1.339 \\
\hline
\end{tabular}

\section{Procedimiento}

La recolección de datos se obtuvo mediante la aplicación de encuestas directamente a los estudiantes mientras realizaban el proceso de matrícula del primer semestre del 2017, respondiendo así a una muestra aleatoria simple sin reposición. Los datos recolectados se analizaron a través de STATA 14, el cual permitió realizar las estadísticas descriptivas (por ejemplo, frecuencias, medias y desviaciones estándar) y el modelo de ecuación estructural informa sobre tres índices estadísticos sobre el grado de ajuste del modelo: bondad de ajuste $\left(\mathrm{X}^{2}\right)$, el error cuadrático medio de aproximación (RMSEA), y el índice de ajuste comparativo (CFI). Donde un valor de cero indica el mejor ajuste para el error cuadrático de aproximación de raíz (RMSEA), (Kline y Santor, 2011). El índice de ajuste comparativo (CFI) es un índice de ajuste incremental que mide la mejora relativa en el ajuste del modelo del investigador sobre el de una línea de base modelo. Para este indicador, típicamente el modelo se considera aceptable cuando, CFI = 1.0. (Kline y Santor, 2011).

\section{Cuestionario}

Para asegurar la validez del cuestionario, se utilizaron preguntas provienen de distintas fuentes previamente utilizadas, La tabla 5 presentan la redacción de las preguntas, las cuales fueron medida la escala de Likert donde 1 es muy en desacuerdo y 5 muy de acuerdo.

Tabla 5: Preguntas del cuestionario.

\begin{tabular}{|c|c|c|c|}
\hline Ítem & $N^{\circ}$ & Pregunta & Fuente \\
\hline \multirow{3}{*}{ IE } & P1 & He pensado muy seriamente en iniciar una empresa. & \multirow{3}{*}{$\begin{array}{l}\text { Francisco } \\
\text { Liñán, et al. } \\
\text { (2011). }\end{array}$} \\
\hline & P2 & Mi objetivo profesional es convertirme en un emprendedor. & \\
\hline & P3 & Haré todo lo posible por iniciar y dirigir mi propia empresa. & \\
\hline \multirow{3}{*}{ A } & P4 & Hacer lo que disfruto es más importante que planificar a largo plazo. & \multirow{3}{*}{$\begin{array}{l}\text { Pingying } \\
\text { Zhang, et al. } \\
\text { (2015). }\end{array}$} \\
\hline & P5 & Hacer lo que disfruto es más importante que evaluar el riesgo a largo plazo. & \\
\hline & P6 & Paso más tiempo haciendo lo que disfruto que planeando a largo plazo. & \\
\hline \multirow{3}{*}{ NS } & P7 & $\begin{array}{l}\text { Creo que los miembros más cercanos de mi familia piensan que debería buscar } \\
\text { comenzar mi propio negocio y convertirme en un emprendedor. }\end{array}$ & \multirow{3}{*}{$\begin{array}{l}\text { Kautonen, } \\
\text { T., et al } \\
(2013) \text {. }\end{array}$} \\
\hline & P8 & $\begin{array}{l}\text { Creo que mis amigos más cercanos piensan que debería buscar mi propio } \\
\text { negocio y convertirme en un emprendedor. }\end{array}$ & \\
\hline & P9 & $\begin{array}{l}\text { Creo que mis colegas (los compañeros de curso) y las personas importantes para } \\
\text { mí piensan que continuar con mi propio negocio y convertirse en emprendedor. }\end{array}$ & \\
\hline \multirow{3}{*}{ PCB } & P10 & Tengo confianza que tendré éxito en la realización de nuevas tareas. & \multirow{3}{*}{$\begin{array}{l}\text { Jintong Tang } \\
\text { (2008). }\end{array}$} \\
\hline & P11 & Tengo confianza que podré alcanzar las metas que yo mismo me fijo. & \\
\hline & P12 & Tengo confianza que tendré éxito cuando enfrente obstáculos. & \\
\hline
\end{tabular}

\section{RESULTADOS Y DISCUSIÓN}

Esta sección presenta los resultados del análisis estadístico, comenzando por la estadística descriptiva y luego el modelo de ecuación estructural. Posteriormente se somete los resultados a una breve discusión en torno al contexto de la intención emprendedora. 


\section{Estadística descriptiva}

La tabla 6 presenta un resumen de las principales estadísticas descriptivas de cada una de las preguntas asociadas a los constructos evaluados. Se ilustra que en general los estudiantes perciben moderadamente a alto en todos los ítems que miden la intención a emprender. Se destaca el hecho que las preguntas asociadas a la actitud tienen un valor medio mayor en comparación con otras cuatro escalas. Además, se informa sobre las cargas factoriales, esta explica el $64,5 \%$ de la varianza de los datos. Así mismo, se determina la validez del instrumento mediante el alfa de Cronbach, esta indica valores mayores o iguales a 0,8 , corroborando la confiabilidad del constructo en la muestra de investigación. De acuerdo con la tabla 7 , se aprecian bajos niveles de correlación (valores entre 0,11 y 0,30 ) entre las variables independientes, estos valores permiten asegurar la independencia de las variables.

Tabla 6: Resumen estadístico descriptivo.

\begin{tabular}{|c|c|c|c|c|c|c|c|}
\hline$N^{\circ}$ Pregunta & Observaciones & Mean & Std Dev & Min & Max & $\begin{array}{c}\text { Carga } \\
\text { Factorial }\end{array}$ & $\begin{array}{c}\text { Cronbach's } \\
\text { alpha }\end{array}$ \\
\hline P1 & 1.339 & 3,666 & 0,975 & 1 & 5 & 0,4356 & \multirow{3}{*}{0,877} \\
\hline P2 & 1.339 & 3,462 & 0,963 & 1 & 5 & 0,5462 & \\
\hline P3 & 1.339 & 2,056 & 1,183 & 1 & 5 & 0,5667 & \\
\hline $\mathrm{P} 4$ & 1.339 & 3,923 & 0,975 & 1 & 5 & 0,4185 & \multirow{3}{*}{0,799} \\
\hline P5 & 1.339 & 2,476 & 1,130 & 1 & 5 & 0,5426 & \\
\hline P6 & 1.339 & 4,187 & 0,937 & 1 & 5 & 0,5945 & \\
\hline P7 & 1.339 & 3,262 & 1,155 & 1 & 5 & 0,2893 & \multirow{3}{*}{0,871} \\
\hline P8 & 1.339 & 2,980 & 1,142 & 1 & 5 & 0,2792 & \\
\hline P9 & 1.339 & 2,976 & 0,981 & 1 & 5 & 0,3516 & \\
\hline $\mathrm{P} 10$ & 1.339 & 2,979 & 0,981 & 1 & 5 & 0,5826 & \multirow{3}{*}{0,886} \\
\hline $\mathrm{P} 11$ & 1.339 & 3,039 & 1,068 & 1 & 5 & 0,6076 & \\
\hline P12 & 1.339 & 2,968 & 1,363 & 1 & 5 & 0,6164 & \\
\hline
\end{tabular}

Tabla 7: Resumen índice de correlación variables independientes.

\begin{tabular}{|c|c|c|c|}
\hline Variable & $A$ & NS & $P C B$ \\
\hline A & 1 & - & - \\
\hline NS & 0,110 & 1 & - \\
\hline PCB & 0,172 & 0,302 & 1 \\
\hline
\end{tabular}

\section{Modelo de ecuación estructural}

A partir del modelo de ecuación estructural, se probó el ajuste del modelo de medición respecto al hipotetizado. Mediante la $X^{2}$, el CFI, el RMSEA y el SRMR, se calculó el ajuste del modelo propuesto. Los resultados del ensayo del modelo indicaron un ajuste aceptable: $X^{2}(66, \mathrm{~N}=1.339)=4935,656, \mathrm{CFI}=0.944$, RMSEA $=0.065$ [90\% IC: $0.000 / 0.072]$, SRMR $=0.039$. Respecto a los efectos calculados los hallazgos mostraron ser significativo y positivos. Se tiene que para la actitud $(A)$ medida según la aversión al riesgo $(b=0,76 ; p<0.01)$, norma subjetiva (NS) $(b=0,17 ; p<0.01)$ y comportamiento del control percibido (PCB) $(b=0,41 ; p<0.01)$ medida en términos de autoeficacia sobre la intención emprendedora.

Tabla 8: Resultados modelo educación estructural.

\begin{tabular}{|c|c|c|c|c|c|c|c|}
\hline Likehood ratio & Population error & \multicolumn{2}{|c|}{ Information criteria } & \multicolumn{2}{c|}{ Base line comparation } & \multicolumn{2}{c|}{ Size of residual } \\
\hline $\mathrm{X}^{2}$ & RMSEA & AIC & BIC & CFI & TLI & SRMR & CD \\
\hline 4935,656 & 0,065 & 38785 & 39004 & 0,944 & 0,923 & 0,039 & 0,944 \\
\hline
\end{tabular}

Tabla 9: Nivel de significancia de las variables independientes.

\begin{tabular}{|c|c|c|c|c|c|c|}
\hline Variable & Coefficient & OIM Std Error & $z$ & $P>/ z /$ & \multicolumn{2}{|c|}{$95 \%$ coef , Interval } \\
\hline A & 0,769 & 0,068 & 11,31 & 0,000 & 0,636 & 0,903 \\
\hline NS & 0,171 & 0,030 & 9,81 & 0,000 & 0,110 & 0,231 \\
\hline PCB & 0,410 & 0,041 & 5,56 & 0,000 & 0,328 & 0,492 \\
\hline
\end{tabular}


Luego, la Figura 2, muestra gráficamente los resultados del modelo de ecuación estructural para la muestra de pregrado. Se verifican las relaciones existentes entre las variables latentes y las observables. En ella se evidencia 12 variables observadas, así también 78 elementos conocidos en la matriz de covarianza, $(12 \mathrm{x}$ $[12+1]) / 2=78)$ y 66 grados de libertad, $(78-12=66)$. Por último, el modelo conceptual se ajusta bien a los datos sobre la intención emprendedora, es decir, se ha comprobado que cuanto mayor es la actitud, la norma subjetiva y el control de la conducta percibida la intención emprendedora aumenta.

\section{DISCUSIÓN FINAL}

En síntesis, a la luz de los resultados obtenidos se confirmar las hipótesis planteadas, es decir las creencias individuales (A), sociales (NS) y motivaciones (PCB) previas sobre el emprendimiento influyen en la intención emprendedora (IE) de los estudiantes. Estos hallazgos, permiten contribuir a la comprensión de las dinámicas del proceso de creación de emprendimientos empresariales basado en la intención emprendedora. Así mismo, se apoya la idea que no necesariamente la presencia de los programas de educación empresarial contribuye a las intenciones para el influir en un emprendimiento, debido a la idea que las acciones emprendedoras requieren del proceso de percepción de las personas (Krueger, 2003), la cual está influenciada por las creencias previas. En efecto, la actitud es el constructo que tiene mayor coeficiente seguido por el control de la conducta percibida. La norma subjetiva es predictor más bajo respecto a las intenciones. Estos resultados son coherentes en significancia y magnitud respecto al meta-análisis realizado por (Armitage y Conner, 2001) para la (TPB).

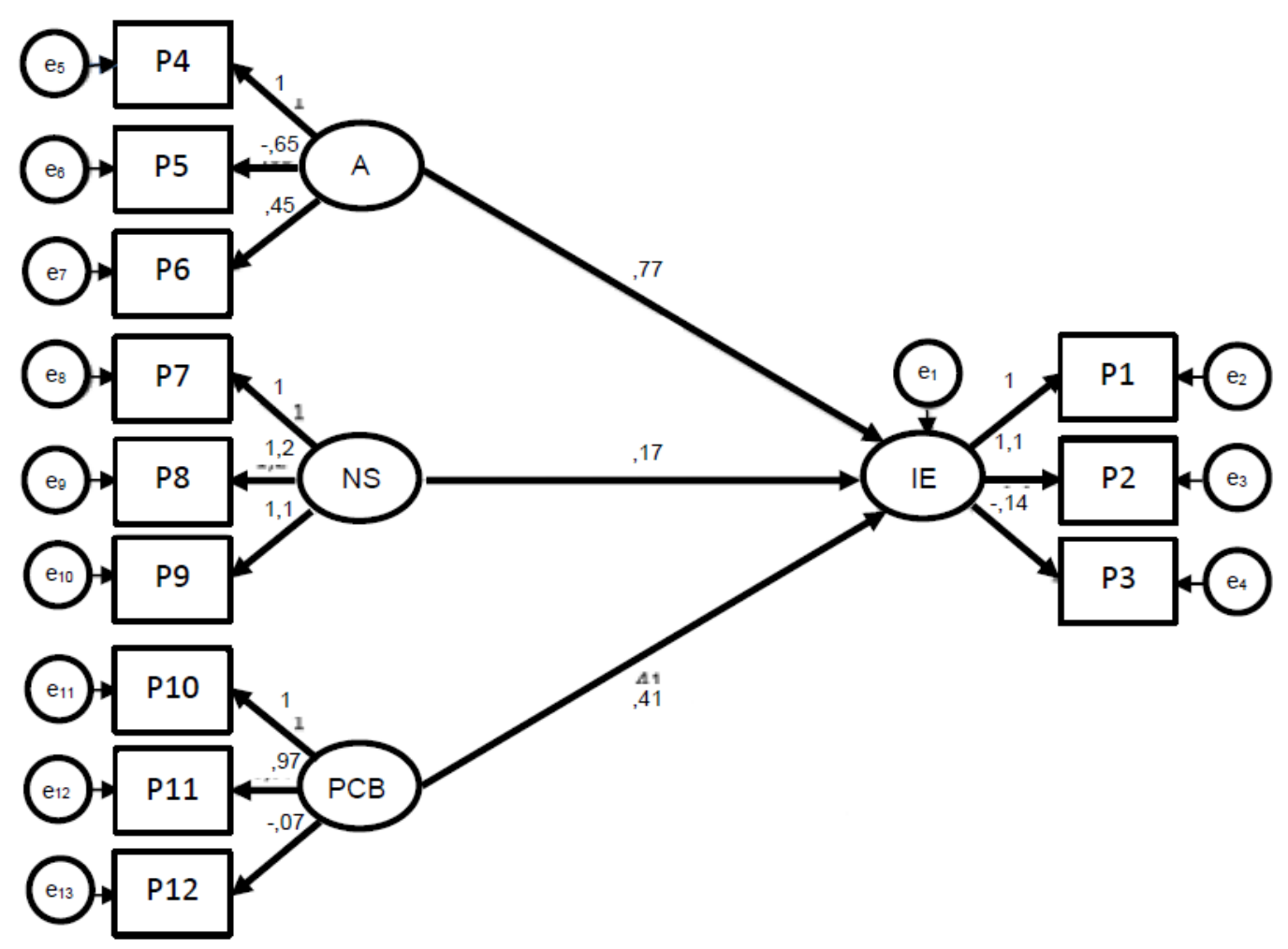

Fig. 2: Resultados para el modelo hipotetizado en ecuaciones estructurales.

Probablemente, ambas magnitudes sean explicadas dado que un importante porcentaje de los alumnos evaluados pertenecen a la generación millennials. Esto quiere decir, por un lado, que los alumnos tienen la impronta de asumir más riesgos debido a que se sienten presionados y con la necesidad de sobresalir y hacer bien los desafíos encomendados y por otro lado valora más su propia creencia respecto a la de otros. Se ha demostrado que los millennials, en comparación con las generaciones anteriores a la misma edad, son más altos en rasgos positivos como la autoestima, asertividad y narcisismo, (Twenge et al., 2008).

Si bien los resultados de la investigación son optimistas, el estudio presenta algunas limitaciones, por ejemplo, la presencia de datos de corte trasversal inhabilita establecer una relación causal, por lo cual se sugiere realizar una medición con datos longitudinales. Otra limitación, basado en la teoría, es la no estimación de la influencia de las variables (PCB) e intención respecto al comportamiento, esto debido a que el diseño fue por conveniencia definida en un tiempo delimitado. 
Adicionalmente, dado que en la investigación se consideraron solo respuestas auto-reportadas de carácter no objetiva, se sugiere realizar un seguimiento para verificar si las intenciones de emprendimiento se concretan en el comportamiento final, esto producto que un $86 \%$ de los estudiantes encuestados se encuentran en los primeros tres años de sus carreras. Esto podría tener consecuencias, de acuerdo con lo planteado por (Kautonen, et al, 2015), quien señala varias explicaciones de por qué las intenciones no se traducen en acciones. Por ejemplo, desde la perspectiva (TPB), la fuerza de la intención impulsa la acción, esto quiere decir que aquellos alumnos con intenciones más débiles son menos propensos a tomar medidas. Las intenciones pueden carecer de estabilidad o elaboración, y tal vez no entusiasmen al estudiante lo suficiente como para conducir a la iniciación de la acción. Otra posibilidad es que las preferencias de algunos estudiantes cambien junto con sus circunstancias personales y profesionales. Por ejemplo, para el caso de los alumnos de los últimos niveles, puede ocurrir que deliberadamente posterguen la decisión a emprender hasta después de haber ganado alguna experiencia, ya sea laboral o personal. Por el contrario, para los alumnos de los primeros niveles, la necesidad académica puede estar por sobre la intención a emprender.

En otras palabras, el individuo puede estar inclinado a dar evaluaciones equivalentemente fuertes, tanto positivas como negativas, hacia un objeto de actitud ante un mismo hecho (Armitage y Conner, 2000), por tanto, una línea de investigación es profundizar el efecto moderador de la ambivalencia actitudinal entre la actitud y la intención emprendedora y entre la intención y el comportamiento. Así también, se sugiere investigar el efecto mediador de la intención empresarial entre las actitudes y el comportamiento.

Finalmente, algunas oportunidades identificadas podrían contribuir a las implicancias prácticas de la investigación, como es el hecho de aprovechar las creencias y motivaciones de los estudiantes respecto a la intención empresarial para ampliar la oferta de cursos relacionado con la educación emprendedora. Por ejemplo, apoyado en la reflexión de (Fayolle y Gailly, 2015), quien señala que los programas de educación empresarial puede aumentar las intenciones y el comportamiento de las personas al influir en los antecedentes de su intenciones de poner en marcha una empresa, los encargados de los procesos instruccionales (en educación) o de capacitación (en empresas u organizaciones públicas) podrían ayudar al diseño de programas más robustos, de manera tal de obtener mejores resultados respecto al desarrollo de competencias que permitan promover el comportamiento emprendedor. De esta forma, converger a que los programas educativos cubran actividades contemporáneas, es decir, creación de startup, avanzar para establecer relación universidad-empresa-entorno, descubrir y resolver problemas (privados y sociales) con recursos limitados. Por lo cual, es primordial fortalecer programas curriculares donde las competencias emprendedoras, traducidas en conocimientos, habilidades, actitudes, normas subjetivas y percibidas adquieran relevación para proveer a los estudiantes, por un lado, la oportunidad de participar en actividades empresariales o en su defecto la simulación de ellas y por otra parte podría ayudar a capacitar a los alumnos a generar, promover e implementar ideas y la afinidad de asumir riesgos.

\section{CONCLUSIONES}

De los resultados mostrados, de su análisis y de su discusión se pueden extraer las siguientes conclusiones sobre la intención emprendedora de los alumnos de pregrado:

1) Se apoya la idea que no necesariamente la presencia de los programas de educación empresarial contribuye a las intenciones para el desarrollo de un emprendimiento;

2) Se refuerza la validez de la (TPB) como el fundamento para explicar la intención del comportamiento;

3) Se determina que las creencias individuales, sociales y motivaciones propias del individuo sobre el emprendimiento pueden influir en la intención emprendedora;

4) Es posible confirmar las hipótesis planteadas, es decir, la actitud, la norma subjetiva y el (PBC) son significativas y están positivamente relacionadas con la intención emprendedora;

5) los resultados permiten reflexionar que el camino más fuerte en el modelo es el que va desde las actitudes hacia la intención emprendedora.

\section{REFERENCIAS}

Alferaih, A., Weight-and Meta-Analysis of Empirical Literature on Entrepreneurship: Towards a Conceptualization of Entrepreneurial Intention and Behavior, doi: 10.1177/1465750317722114, The International Journal of Entrepreneurship and Innovation, 18(3), 195-209 (2017)

Ajzen, I., The Theory of Planned Behavior, doi: 10.1016/0749-5978(91)90020-T 50(2), Organizational Behavior and Human Decision Processes, 179-211 (1991)

Al-Shammarim, M., Entrepreneurial Intention of Private University Students in the Kingdom of Bahrain, doi:10.1108/IJIS06-2017-0058, International Journal of Innovation Science (2018) 
Armitage, C.J. y M. Conner, Efficacy of the Theory of Planned Behaviour: A Meta-Analytic Review, doi:10.1348/014466601164939, British Journal of Social Psychology, 40(4), 471-499 (2001)

Armitage, C. J. y M. Conner, Attitudinal Ambivalence: A test of Three Key Hypotheses, doi: 10.1177/0146167200263009, Personality and Social Psychology Bulletin, 26(11), 1421-1432 (2000)

Bae, T.J., S. Qian, C. Miao y J.O. Fiet, The Relationship between Entrepreneurship Education and Entrepreneurial Intentions: A meta-Analytic Review, doi: 10.1111/etap.12095, Entrepreneurship Theory and Practice, 38(2), 217-254 (2014)

Entrialgo, M. y V. Iglesias, The Moderating Role of Entrepreneurship Education on the Antecedents of Entrepreneurial Intention, doi: 10.1007/s11365-016-0389-4, International Entrepreneurship and Management Journal, 12(4), 1209-1232 (2016)

Fayolle, A. y B., Gailly, The Impact of Entrepreneurship Education on Entrepreneurial Attitudes and Intention: Hysteresis and Persistence, doi:10.1111/jsbm.12065, Journal of Small Business Management, 53(1), 75-93 (2015)

Fietze, S. y B. Boyd, Entrepreneurial Intention of Danish Students: A Correspondence Analysis, doi: 10.1108/IJEBR-082016-0241, International Journal of Entrepreneurial Behavior y Research, 23(4), 656-672 (2017)

Iglesias-Sánchez, P. P., C. Jambrino-Maldonado, A. P. Velasco y H. Kokash, Impact of Entrepreneurship Programmes on University Students, doi:10.1108/ET-01-2015-0004, Education + Training, 58(2), 209-228 (2016)

Kautonen, T., M. Van Gelderen y M. Fink, Robustness of the Theory of Planned Behavior in Predicting Entrepreneurial Intentions and Actions, doi:10.1111/etap.12056, Entrepreneurship Theory and Practice, 39(3), 655-674 (2015)

Kautonen, T., M. Van Gelderen y E.T. Tornikoski, Predicting Entrepreneurial Behaviour: A Test of the Theory of Planned Behavior, doi:10.1080/00036846.2011.610750, Applied Economics, 45(6), 697-707 (2013)

Kline, R. B. y D. A, Santor, Principles y Practice of Structural Equation Modelling, $3^{\text {ra }}$ Ed., The Guilford Press, 50-51, New York America, United States of America (2011)

Kirkley, W. W., Entrepreneurial Behaviour: The Role of Values, doi: 10.1108/IJEBR-02-2015-0042, International Journal of Entrepreneurial Behavior y Research, 22(3), 290-328 (2016)

Krauss, C. y otros cuatro autores, Intención Emprendedora en Estudiantes de Ciencias de la Salud de la Universidad Católica del Uruguay, doi: 10.22235/ech.v7i1.1541, Enfermería: Cuidados Humanizados, 7(1), 43-56 (2018)

Krueger, N.F., M.D. Reilly y A.L. Carsrud, Competing Models of Entrepreneurial Intentions, doi: 10.1016/S08839026(98)00033-0, Journal of Business Venturing, 15(5-6), 411-432 (2000)

Krueger, N.F., The Cognitive Psychology of Entrepreneurship. In Handbook of Entrepreneurship Research, 105-140, Springer, Boston, United States of America (2003)

Laguía, A., J. A. Moriano, F. Molero y J. A. Gámez, Validación del Cuestionario de Intención Emprendedora en una Muestra de Estudiantes Universitarios de Colombia, doi: 10.11144/Javeriana.upsy16-1.vcie, Universitas Psychologica, 16(1), 60$73(2017)$

Lima, E., R.M. Lopes, V. Nassif y D. Silva, Opportunities to Improve Entrepreneurship Education: Contributions Considering Brazilian Challenges, doi:10.1111/jsbm.12110, Journal of Small Business Management, 53(4), 1033-1051 (2015)

Liñán, F., J. C. Rodríguez-Cohard y J.M. Rueda-Cantuche, Factors Affecting Entrepreneurial Intention Levels: a Role for Education, doi: 10.1007/s11365-010-0154-z, International Entrepreneurship and Management Journal, 7(2), 195-218 (2011)

Osorio, F. F. y J. C. Londoño Roldán, Intención Emprendedora de Estudiantes de Educación Media: Extendiendo la Teoría de Comportamiento Planificado Mediante el Efecto Exposición, doi: 10.11144/Javeriana.cao28-51.ieee, Cuadernos de Administración, 28(51) (2015)

Rauch, A. y W. Hulsink, Putting Entrepreneurship Education Where the Intention to Act Lies: An investigation Into the Impact of Entrepreneurship Education on Entrepreneurial Behavior, doi:10.5465/amle.2012.0293, Academy of Management Learning y Education, 14(2), 187-204 (2015)

Schlaegel, C. y M. Koenig, Determinants of Entrepreneurial Intent: a Meta-Analytic Test and Integration of Competing Models, doi:10.1111/etap.12087, Entrepreneurship Theory and Practice, 38(2), 291-332 (2014)

Shane, S. y S. Venkataraman, The Promise of Entrepreneurship as a Field of Research, Academy of Management Review, 25 (1), 217-226 (2000)

Sheeran, P., Intention-Behavior Relations: A Conceptual and Empirical Review, doi: 10.1080/14792772143000003, European Review of Social Psychology, 12(1), 1-36 (2002)

Sitaridis, I. y F. Kitsios, Entrepreneurial Intentions of Information Technology Students: The Theory of Planned Behaviour, the Role of Gender and Education, doi:10.1504/JIBED.2017.085508, Journal for International Business and Entrepreneurship Development, 10(3), 316-335 (2017)

Solesvik, M., P. Westhead y H. Matlay, Cultural Factors and Entrepreneurial Intention: The Role of Entrepreneurship Education, doi: 10.1108/ET-07-2014-0075, Education+ Training, 56(8/9), 680-696 (2014)

Soria-Barreto, K., S. Zúñiga-Jara y S. Ruiz-Campo, Educación e Intención Emprendedora en Estudiantes Universitarios: un Caso de Estudio, doi: 10.4067/S0718-50062016000100004, Formación Universitaria, 9(1), 25-34 (2016) 
Tang, J., Environmental Munificence for Entrepreneurs: Entrepreneurial Alertness and Commitment, doi:10.1108/13552550810874664, International Journal of Entrepreneurial Behavior y Research, 14(3), 128-151 (2008)

Tarapuez, E., M.D. García y N. Castellano, Aspectos Socioeconómicos e Intención Emprendedora en Estudiantes Universitarios del Quindío (Colombia), doi: 10.15446/innovar.v28n67.68618, Innovar: Revista de Ciencias Administrativas y Sociales, 28(67), 123-135 (2018)

Tarapuez-Chamorro, E., Las dimensiones Culturales de Geert Hofstede y la Intención Emprendedora en Estudiantes Universitarios del Departamento del Quindío (Colombia), doi: 10.14482/pege.41.9699, Pensamiento y Gestión, (41) (2016)

Teixeira, S.J., C.M.L., Casteleiro, R. Rodrigues y M. Guerra, Entrepreneurial Intentions and Entrepreneurship in European Countries, doi: 10.1108/IJIS-07-2017-0062, International Journal of Innovation Science (2018)

Twenge, J.M. y otros cuatro autores, Egos Inflating Over Time: A Crosstemporal Meta-Analysis of the Narcissistic Personality Inventory, doi:10.1111/j.1467-6494.2008.00507.x, Journal of Personality, 76(4), 875-901 (2008)

Thomas, A., R. Passaro y G. Scandurra, The Perception of the Contextual Factors as Predictor of Entrepreneurial Intent: Evidences from an Empirical Survey, doi: 10.1142/S0218495814500162, J. of Enterprising Culture, 22 (4), 375-400 (2014)

Westhead, P., D.J. Storey y F. Martin, Outcomes Reported by Students Who Participated in the 1994 Shell Technology Enterprise Programme, doi:10.1080/08985620010018273, Entrepreneurship and Regional Development, 13, 163-85 (2001)

Yukongdi, V. y N.Z. Lopa, Entrepreneurial Intention: A Study of Individual, Situational and Gender Differences, doi:10.1108/JSBED-10-2016-0168, Journal of Small Business and Enterprise Development, 24(2), 333-352 (2017)

Zhang, P., D. D., Wang y C.L. Owen, A Study of Entrepreneurial Intention of University Students, doi:10.1515/erj-20140004, Entrepreneurship Research Journal, 5(1), 61-82 (2015) 
